Published in final edited form as:

Dig Liver Dis. 2018 August ; 50(8): 804-811. doi:10.1016/j.dld.2018.03.021.

\title{
Gene expression-phenotype associations in adults with eosinophilic esophagitis
}

\author{
Evan S. Dellon, MD MPH ${ }^{1,{ }^{*}}$, Sara R. Selitsky, PhD ${ }^{2,{ }^{*}, \text { Robert M. Genta, MD }}{ }^{3,4}$, Richard H. \\ Lash, $\mathbf{M D}^{3}$, and Joel S. Parker, PhD ${ }^{2}$ \\ ${ }^{1}$ Center for Esophageal Diseases and Swallowing, Division of Gastroenterology and Hepatology, \\ Department of Medicine, University of North Carolina, Chapel Hill, NC \\ ${ }^{2}$ Department of Genetics, University of Chapel Hill, NC \\ ${ }^{3}$ Miraca Life Sciences Research Institute, Irving, TX \\ ${ }^{4}$ Dallas Veterans Affairs Medical Center, University of Texas Southwestern Medical Center, \\ Dallas, TX
}

\begin{abstract}
Background-Gene expression patterns have not been extensively examined in the context of clinical features of eosinophilic esophagitis (EoE).

Aims-To assess whether gene expression is associated with clinically defined phenotypes in adults with EoE.

Methods-This was an analysis of prospectively collected esophageal biopsies in newly diagnosed EoE patients. We determined differential gene expression with a 94 gene panel in relation to clinical features and phenotypes. These included: endoscopic findings of esophageal rings, stricture, narrowing, linear furrows, exudates, edema, and dilation; an allergic phenotype; an inflammatory phenotype, and a fibrostenotic phenotype.
\end{abstract}

Results-In 89 EoE cases analyzed, patients with exudates on endoscopy had multiple differences in gene expression compared to patients without exudates, though patients with

Corresponding Author: Evan S. Dellon MD, MPH, CB\#7080, Bioinformatics Building, 130 Mason Farm Rd., UNC-CH, Chapel Hill, NC 27599-7080, Phone: (919) 966-2513, Fax: (919) 843-2508 edellon@med.unc.edu.

co-first authors

Publisher's Disclaimer: This is a PDF file of an unedited manuscript that has been accepted for publication. As a service to our customers we are providing this early version of the manuscript. The manuscript will undergo copyediting, typesetting, and review of the resulting proof before it is published in its final citable form. Please note that during the production process errors may be discovered which could affect the content, and all legal disclaimers that apply to the journal pertain.

Author contributions:

Dellon: Project conception/design; obtained funding; data analysis/interpretation; drafting of the article; critical revision; approved final draft

Selitsky: Data analysis/interpretation; critical revision; approved final draft

Genta: Data interpretation; critical revision; approved final draft

Lash: Data interpretation; critical revision; approved final draft

Parker: Data analysis/interpretation; critical revision; approved final draft

Conflict of Interest Statement

Disclosures: Drs. Genta, and Lash are employees of Miraca Life Sciences. The other authors have no potential conflicts of interest pertaining to this study. 
exudates also had higher eosinophil counts (172 vs 106 eos/hpf; $\mathrm{p}=0.01$ ). Genes associated with esophageal narrowing included CCL26 (q-value=0.028), ALOX15 ( $\mathrm{q}=0.011)$, GRK5 ( $\mathrm{q}=0.029)$, CPA3 ( $\mathrm{q}=0.012$ ), and TRIM2 ( $\mathrm{q}=0.0027)$. TRIM2 was also associated with the fibrostenotic phenotype $(\mathrm{q}=0.0051)$. No genes were associated with the inflammatory or atopic phenotypes, or with dilation.

Conclusions-Multiple genes are associated with exudates, possibly related to higher eosinophil counts. However, a number of genes, including those related to both inflammation and remodelling, are associated with esophageal narrowing. In particular, TRIM2 is associated with clinical fibrotic phenotypes.

\section{Keywords}

fibrostenosis; inflammation; endoscopy; biopsy; RNA

\section{Introduction}

Eosinophilic esophagitis is a Th2-mediated allergen/immune-mediated esophageal disorder. $[1,2]$ Since its description in the mid-1990s, there has been rapid understanding of EoE pathogenesis. In particular, the genetic underpinnings of EoE are beginning to be elucidated. [3] Landmark work by Blanchard, Rothenberg, and colleagues described a set of genes, termed the "EoE transcriptome", that are differentially expressed in EoE patients compared to those with gastroesophageal reflux disease (GERD) and normal controls. [4, 5] Recent work has identified a subset of genes that are highly discriminatory for diagnosis of EoE compared to non-EoE controls,[6] and this same gene set also appears to be responsive, normalizing after successful treatment.[7] In addition, a series of genome-wide association studies (GWAS) have identified several single nucleotide polymorphisms (SNP) that are highly associated with EoE,[8-10] and may increase EoE risk.[11]

In addition to genetics, there has been recognition of different clinical phenotypes in EoE. [12-15] Perhaps the most important one categorizes patients into either an inflammatorypredominant appearance in the esophagus (endoscopic findings of edema, exudates, and linear furrows) or a fibrostenotic-predominant appearance (esophageal strictures or narrowing).[12,16] Other phenotypes can include atopic vs non-atopic, male vs female, white vs non-white, adult vs pediatric, treatment responders vs non-responders, and connective tissue disease-associated.[14, 17] However, associations between genetics and phenotypes have not been extensively examined in EoE. Initial investigations have shown a SNP in transforming growth factor beta-1 (TGF- $\beta 1$ ) may be associated with treatment response,[18] that gene expression related to mast cells correlated with dysphagia measured by a pediatric symptom score,[19] and that expression of some genes may be associated with steroid response.[20]

Because there have been no studies specifically assessing the relationship between genotype and clinical phenotype, the aim of this study was to assess whether gene expression is associated with clinically defined phenotypes in adults with EoE. We hypothesized that expression of genes related to epithelial integrity and remodelling would be associated with 
a fibrostenotic phenotype, while expression of genes related to Th2 cytokines would be associated with an atopic phenotype.

\section{Materials and Methods}

\section{Study design, patients, and clinical data collection}

We conducted an analysis of biopsy samples and data collected during a prospective cohort study of EoE. The parent study, details of which have been described previously,[21-24] enrolled adults ( $\geq 18$ years) undergoing upper endoscopy at University of North Carolina for symptoms of dysphagia, heartburn, refractory reflux, or other upper gastrointestinal symptoms. They were excluded if they had a known (prevalent) diagnosis of EoE or eosinophilic gastroenteritis, acute GI bleeding, active anticoagulation, known esophageal cancer, prior esophageal surgery, known esophageal varices, or medical instability or multiple comorbidities precluding enrollment in the clinical opinion of the endoscopist. Informed consent, including consent for future use of stored biopsy specimens, was obtained prior to the endoscopy. This study was approved by the UNC Institutional Review Board.

Only incident cases of EoE, with baseline (pre-steroid or dietary treatment) samples and data available, were included in the present study. EoE was diagnosed as per 2011 consensus guidelines.[1] Specifically, cases were had at least one symptom of esophageal dysfunction, $\geq 15 \mathrm{eos} / \mathrm{hpf}$ on esophageal biopsy after an 8-week proton pump inhibitor trial (20-40 mg twice daily of any of the available agents, prescribed at the discretion of the clinician), and exclusion of other causes of esophageal eosinophilia. Of note, subjects with proton pump inhibitor-responsive esophageal eosinophilia (PPI-REE) were excluded from the present analysis because we did not have sufficient pre-PPI treatment banked samples available to conduct the analysis, and it has previously been shown that gene expression profiles in this subgroup normalize after PPI administration,[25] so post-PPI samples would not be appropriate to address to the study question.

Demographics, clinical, and endoscopic data were recorded prospectively using standardized case report forms. During the endoscopy, research protocol esophageal biopsies were obtained (two from the proximal, one from the middle, and two from the distal esophagus) in order to determine tissue eosinophil counts and ensure EoE diagnostic sensitivity.[26, 27] We also collected research-protocol gastric and duodenal biopsies to exclude concomitant eosinophilic gastroenteritis. After masking the slides as to clinical status, peak esophageal eosinophil counts were determined based on our previously validated methodology.[28, 29]

\section{Sample collection, RNA extraction, and gene expression}

In addition to the biopsies that were obtained for histologic analysis, additional esophageal biopsy samples were collected, labeled with a de-identified study number, masked as to clinical status, and stored at $-80{ }^{\circ} \mathrm{C}$ RNA-later (Life Technologies/Thermo-Fisher Scientific, Grand Island, NY) for future use. Samples remained frozen until study enrollment was complete and we were ready to perform the present analysis. At that time, we retrieved a single RNA-later-preserved biopsy from the mid-esophagus $(10 \mathrm{~cm}$ above the gastroesophageal junction) for gene expression determination. The rationale for using a 
single mid-esophageal biopsy was based on our prior work showing that gene expression in EoE was similar throughout the esophagus.[30]

Gene expression testing was performed at Miraca Life Sciences (Phoenix, AZ) as previously described.[7] After thawing and tissue homogenization, RNA was extracted using the RNeasy Mini Extraction Kit (Qiagen, Valencia, CA) per the manufacturer's instructions. The concentration was measured using spectrophotometry (NanoDrop, Wilmington, DE) with $16.5 \mathrm{ng} / \mu \mathrm{l}$ of RNA for a total of $500 \mathrm{ng}$ considered acceptable. cDNA synthesis was carried out using the iScript cDNA Synthesis Kit (Bio-Rad, Hercules, CA) with PCR performed on ABI 9700 (Applied Biosystems, Foster City, CA). The cDNA and TaqMan Universal Master Mix II, no UNG (Life Technologies) were loaded onto custom Taqman TLDA cards containing preloaded Taqman gene expression assays in a 384-well format. This consisted of the 94 gene panel that was previously developed for EoE and 2 housekeeping genes (GAPDH and 18S). PCR was performed on Quant Studio 7 (Life Technologies) to determine the gene expression levels measured as threshold cycles $(\mathrm{Ct})$. Samples with a GAPDH value of $<30 \mathrm{Ct}$ were considered acceptable. A gene expression summary score was calculated using a previously established algorithm.[6] Specifically, the $\mathrm{Ct}$ value of the housekeeping gene was subtracted from the $\mathrm{Ct}$ value of each gene of interest to acquire the $\Delta \mathrm{CT}$, and then the absolute values of the normalized gene $\mathrm{Ct}$ values were summed for each gene in the gene expression panel. In general, a gene score below 333 is characteristic of EoE, with lower scores being more reflective of a diagnosis of EoE.[6]

\section{Clinical phenotype definition and statistical analysis}

Clinical findings and phenotypes for analysis were defined as follows. Endoscopic findings of interest were reported by the endoscopic and included: esophageal rings, stricture, narrowing, furrows, exudates, and edema, as well as whether esophageal dilation was performed. Strictures were defined as the presence of a focal area of constriction in the esophagus. Narrowing was defined as an area where the lumen had a smaller caliber, but was more diffuse than what would be expected for a focal stricture. The fibrostenotic phenotype was defined as the presence of stricture, narrowing, or dilation, regardless of other endoscopic findings. A "severe" fibrotic phenotype was defined if there was a focal stricture or narrowing with an esophageal diameter measured as $10 \mathrm{~mm}$ (such that a standard adult upper endoscope used in our procedures unit would not pass). The inflammatory phenotype was defined as the presence of exudates, edema, or furrows, in the absence of dilation being performed, and regardless of other endoscopic findings. An inflammatory "only" phenotype was defined as having exudates, edema, or furrows without other endoscopic findings. The allergic phenotype was defined as the presence of any atopic condition.

We used summary statistics to describe the clinical features of the EoE cases. Among the EoE cases, the mean summary gene score was compared for the patients with and without each endoscopic finding or phenotype using a two-sample t-test. We then determined differential gene expression for each endoscopic finding or phenotype using the MannWhitney $\mathrm{U}$ test with a false discovery rate, calculated using the Benjamini-Hochberg procedure, of 0.1. Differential gene expression was then plotted on a heat map. Finally, we performed unsupervised consensus clustering with the R package, ConsensusClusterPlus 
(parameters $\operatorname{maxK}=6$, reps $=50$, pItem $=0.8$ ) using all of the genes.[31]

ConsensusClusterPlus provides stable clusters by iteratively clustering a random subset of samples a given amount of times and then using the consensus results. This yielded two distinct clusters. We then compared clinical, endoscopic, and histologic features between each of these clusters, to determine the clinical phenotypic correlates of the gene expression clustering.

\section{Results}

\section{Patient characteristics and phenotypes}

A total of 89 incident cases of EoE were included in this analysis. Full clinical details for this population have been previously described.[7] In brief, the mean age was $37 \pm 13$ years, $58 \%$ were men, $94 \%$ were white, and nearly all (99\%) had dysphagia-predominant symptoms. $74 \%$ had at least one atopic condition (29\% asthma, 65\% allergic rhinitis/ sinusitis, $8 \%$ eczema, $38 \%$ food allergies) and were categorized into the allergic phenotype.

On endoscopy, typical EoE findings were common, including $80 \%$ with rings, $25 \%$ with a focal stricture, $36 \%$ with narrowing, $87 \%$ with furrows, $47 \%$ with exudates, and $56 \%$ with edema. Dilation was performed in $29(33 \%)$. A total of 49 patients $(55 \%)$ were categorized into the fibrostenotic phenotype, with $13(15 \%)$ meeting criteria for "severe" fibrosis. There were 57 (64\%) who met the definition for the inflammatory phenotype, but only $6(7 \%)$ with inflammation-only findings. The average peak eosinophil count at baseline for overall study population was $137 \pm 124 \mathrm{eos} / \mathrm{hpf}$.

\section{Gene expression-phenotype associations}

Summary gene scores differed with the presence of several individual endoscopic findings, but did not differ substantially for the atopic phenotypes or endoscopically defined inflammatory or fibrostenotic phenotypes (Figure 1). For example, EoE patients with esophageal narrowing (149 vs 228; $\mathrm{p}=0.009$ ), linear furrows (186 vs $287 ; \mathrm{p}=0.02$ ), exudates (129 vs $263: \mathrm{p}<0.001)$, or edema (171 vs $236 ; \mathrm{p}=0.03$ ) had significantly lower gene scores compared to those without these findings. There was also a trend for lower scores in the presence of rings ( 187 vs $247 ; \mathrm{p}=0.10)$.

Differential gene expression for individual genes is shown relation to clinical phenotypes and endoscopic findings as a heat map in Figure 2. Patients with exudates on endoscopy had differences in gene expression for the majority of genes compared to patients without exudates, and many of these differences were at the Benjamini-Hochberg corrected q-value $<0.025$ level. Of note, patients with exudates also had higher eosinophil counts than those without exudates $(172 \pm 141$ eos/hpf vs $106 \pm 99 ; \mathrm{p}=0.01)$. There was a trend towards increased expression of the gene TRIM2 with the presence of both rings and stricture (qvalues $=0.086$ and 0.091 , respectively). Several genes had decreased expression and were associated with narrowing (Figure 3A), including CCL26 (q-value $=0.028)$, ALOX15 (qvalue $=0.011)$, GRK5 (q-value $=0.029)$, APOBEC3A $(q-$ value $=0.076)$, HPGDS $(q-$ value $=$ $0.057)$, CPA3 (q-value $=0.012)$, POSTN (qvalue $=0.058)$, and TRIM2 $(\mathrm{q}$-value $=0.0027)$. Increased expression of TRIM2 was also associated with the fibrostenotic phenotype and 
severe fibrosis (q-value $=0.0051$ and 0.027 , respectively) (Figure 3B). IL4 and CFI were associated with furrows (q-value $=0.043$ and 0.052 , respectively), and there was a trend for an association between CRISP2 (q-value $=0.058$ ), CRISP3 (q-value $=0.097$ ), and TSPAN12 (q-value $=0.087$ ) and edema. We did not identify any genes that were clearly associated with either the inflammatory or atopic phenotypes, or with esophageal dilation.

\section{Unsupervised clustering of eosinophilic esophagitis}

We performed unsupervised clustering of the EoE samples using the gene expression data, and identified two independent clusters (Figure 4). The principal component analysis plot shows segregation in PC1 (Figure 4A). The heat map of all genes in this gene expression panel show that the immune related genes are higher in cluster 2 and cell proliferation and epithelial makers are higher in cluster 1 (Figure 4B).

In terms of clinical features, cluster 1 had a higher proportion of women than cluster 2 (49\% vs $34 \%)$, but this was not significant $(\mathrm{p}=0.16)$ (Table 1). There were also no differences in other demographic, atopic features, or endoscopically defined phenotypes. However, there were a number of individual endoscopic correlates. EoE cases in cluster 1 were more likely to have exudates ( $69 \%$ vs $25 \%$; $p<0.001$ ), edema ( $67 \%$ vs $45 \%$; $p=0.04)$, and crêpe-paper mucosa $(11 \%$ vs $0 \%$; $\mathrm{p}=0.02)$. There were also histologic differences, with EoE cases in cluster 1 having more eosinophil degranulation (100\% vs 91\%; $\mathrm{p}=0.04)$, eosinophilic microabscesses ( $80 \%$ vs $45 \%$; $\mathrm{p}<0.001)$, and basal layer hyperplasia $(53 \%$ vs $33 \%$; $\mathrm{p}=$ 0.02 ), as compared to cluster 2 (Table 1).

\section{Discussion}

While knowledge about the role of genetics in the pathogenesis of EoE has rapidly increased,[3] there is a relative paucity of data examining clinical correlates of gene expression. We conducted an analysis of prospectively collected and banked esophageal biopsies in order to assess whether gene expression is associated with clinically defined phenotypes in adults with EoE, a study that is the first of its kind. Overall, we found a number of gene expression-clinical correlations of note. First, mean gene scores were lower, indicating more active EoE with more differentially expressed genes, in patients with the individual endoscopic findings of exudates, furrows, edema, and narrowing. Second, there was significant differential gene expression in multiple genes in EoE patients with exudates, and this could be related to substantially higher eosinophil counts in those patients. There were also a number of genes, including those related to both inflammation and remodelling, associated with esophageal narrowing. In particular, the gene TRIM2 was associated with clinical fibrotic phenotypes. Interestingly, gene expression was not associated with the atopic or inflammatory phenotypes. Finally, an unsupervised principle component analysis revealed that our EoE cases could be segregated into two populations based on gene expression, with features of one cluster including higher esophageal eosinophil counts and a larger proportion of exudates, edema, and crêpe-paper mucosa. We did not find specific gene expression patterns in patients with and without an atopic phenotype, or in those with and without an inflammatory phenotype. 
Our study provides new information about patients with fibrostenotic remodelling of the esophagus in EoE, which has previously been felt to be a consequence of unchecked inflammation during a long disease duration prior to diagnosis or treatment.[12, 32-35] These patients have also been noted to have a less compliant esophagus which raises the risk for food bolus impaction, [36, 37] and may constitute a population that is more refractory to topical steroid treatment.[17] However, the reason why some patients develop a more fibrotic phenotype has not been clear. Our data may provide some clues. In patients with esophageal narrowing, a number of genes have increased expression, including CCL26, ALOX15, GRK5, APOBEC3A, HPGDS, CPA3, POSTN, and TRIM2. These genes are involved in inflammatory processes, for example CCL26 (eotaxin-3), and ALOX15 (arachidonate 15-lipoxygenase), as well as mast cell function (CPA; carboxypeptidase A3) and remodeling (POSTN; periostin).[3-5, 38] The gene TRIM2 (tripartite motif containing 2 protein) may be of special interest, as this was also associated with rings, stricture, the fibrostenotic phenotype, and "severe" fibrosis. TRIM2 is an E3 ubiquitin ligase that has been implicated in neuronal development and function[39-41] as well as tissue homeostasis related to ischemia.[42] Mutations in this gene have been associated with axonal neuropathy and Alzheimer's disease.[43, 44] However, the function of TRIM2 in EoE or in fibrosis has not been explored specifically, and this should be a future direction of mechanistic research. Interestingly, we did not find evidence of differential expression of desmoglein (DSG), which has previously been linked to dysregulation of the epithelial barrier.[45]

We also found strong trends towards associations between IL4, CFI, and furrows, and between CRISP2, CRISP3, and TSPAN12 and edema, but the mechanisms behind these associations are unknown and would need to be elucidated in future research. IL4 codes for interleukin 4, a key type 2 cytokine that has been implicated in EoE pathogenesis,[3] and recent phase 2 data support blocking the IL-4 receptor as a promising treatment strategy for EoE.[46] CFI codes for complement factor I, a serine protease involved in complement cascade regulation, but which has not been associated with atopy.[47] CRISP2 and -3 code for cysteine-rich secretory proteins 2 and 3 which are thought to be involved in epithelial processes, and TSPAN12 codes for tetraspanin-12, a regulator of cell surface signal transduction, but the exact role of these genes in EoE pathogenesis is not known.[3, 6]

There are some data in the literature that provide additional context for our findings. In the initial description of the EoE transcriptome, Blanchard and colleagues showed similar gene expression profiles between males and females with EoE, and allergen-sensitized and nonsensitized EoE patients.[4] This latter finding is consistent with our finding of no differences in expression for the atopic phenotype. In a related study, similar expression profiles were also seen in EoE patients with a family history of EoE and those without a family history. [48] Martin and colleagues showed that gene expression related to mast cells correlated with dysphagia as measured by a validated pediatric symptom score.[19] This included the gene CPA3 which we observed was linked to esophageal narrowing in the present study. In a recent abstract, a TSLP risk allele was associated with having multiple EoE food triggers, and could impact on treatment recommendations.[49] Two published studies have also looked at the impact of genetic variability on treatment outcomes. Aceves and colleagues demonstrated that children with EoE more likely to be responders treatment if they had a SNP (CC genotype) at the -509 position in the TGF- $\beta_{1}$ promoter.[18] Though this finding 
has not yet been replicated, Butz and colleagues found several differentially expressed genes were associated with treatment response in a sub-analysis from a randomized clinical trial of fluticasone.[20] Some of these, including ALOX15, CCL26, APOBEC3A, and POSTN, overlap with findings from our study, but we could not specifically assess treatment response since we were examining baseline features only. However, we have presented abstract data where the gene panel did not appear to predict histologic response to treatment.[50]

There are limitations of this study to acknowledge. First, this was a single center study of adults only, so it is difficult to compare directly to prior gene expression results that have been primarily in pediatric EoE populations. Second, the endoscopic findings, and in particular the fibrostenotic findings were determined by the performing endoscopist, so there may be some variability in reporting. Third, the gene expression analysis was limited to the 94 genes in the preexisting panel, and it is possible that additional differences might be noted with whole genome RNA sequencing. However, a full RNA sequencing project was beyond the scope of the current study. Last, the study design only allows determination of associations, so we are unable to know if these expression differences are a result of the disease or a cause of the phenotype, and mechanistic analysis would be required to understand the role of genes such as TRIM2 in the pathogenesis of EoE. However, there are a number of strengths to the study that lend validity to the results. This study used specimens that were collected during a rigorously conducted prospective cohort study of EoE. In addition, all of the samples were obtained from newly diagnosed EoE cases prior to either topical steroid or dietary elimination treatment. Specimens were handled and stored uniformly, and extensive clinical data were available to allow full clinical, endoscopic, and histologic characterization of the EoE cases. This study is also the first of its kind to extensively evaluate the association between gene expression and clinical/endoscopic phenotypes.

In conclusion, in this study of prospectively collected samples, we found that multiple genes in the 94 gene EoE diagnostic panel were associated with endoscopic findings of exudates, possibly related to significantly higher eosinophil counts in these patients. We noted a more focused number of genes, including those related to both inflammation and remodelling, were associated with esophageal narrowing. In particular, TRIM2 was associated with clinical fibrotic phenotypes. These results provide initial insight into genetic determinants of different presentations of EoE, and provide a foundation for future mechanistic studies. The future studies should also focus on assessing phenotypes over time and predicting progression of phenotypes.

\section{Acknowledgments}

Grant support: This work was supported, in part, by NIH Awards K23DK090073 (ESD) and an investigatorinitiated research grant from Miraca Life Sciences (ESD), and uses resources from the UNC Center for GI Biology and Disease (P30DK34987) and the UNC Translational Pathology lab (P30CA016086).

\section{References}

1. Liacouras CA, Furuta GT, Hirano I, et al. Eosinophilic esophagitis: Updated consensus recommendations for children and adults. J Allergy Clin Immunol. 2011; 128:3-20.e6. [PubMed: 21477849] 
2. Dellon ES, Gonsalves N, Hirano I, et al. ACG Clinical Guideline: Evidence based approach to the diagnosis and management of esophageal eosinophilia and eosinophilic esophagitis. Am J Gastroenterol. 2013; 108:679-92. [PubMed: 23567357]

3. Rothenberg ME. Molecular, Genetic, and Cellular Bases for Treating Eosinophilic Esophagitis. Gastroenterology. 2015; 148:1143-57. [PubMed: 25666870]

4. Blanchard C, Wang N, Stringer KF, et al. Eotaxin-3 and a uniquely conserved gene-expression profile in eosinophilic esophagitis. J Clin Invest. 2006; 116:536-47. [PubMed: 16453027]

5. Sherrill JD, Kiran KC, Blanchard C, et al. Analysis and expansion of the eosinophilic esophagitis transcriptome by RNA sequencing. Genes Immun. 2014; 15:361-9. [PubMed: 24920534]

6. Wen T, Stucke EM, Grotjan TM, et al. Molecular diagnosis of eosinophilic esophagitis by gene expression profiling. Gastroenterology. 2013; 145:1289-99. [PubMed: 23978633]

7. Dellon ES, Veerappan R, Selitsky SR, et al. A Gene Expression Panel is Accurate for Diagnosis and Monitoring Treatment of Eosinophilic Esophagitis in Adults. Clin Transl Gastroenterol. 2017; 8:e74. [PubMed: 28181994]

8. Rothenberg ME, Spergel JM, Sherrill JD, et al. Common variants at $5 \mathrm{q} 22$ associate with pediatric eosinophilic esophagitis. Nat Genet. 2010; 42:289-91. [PubMed: 20208534]

9. Kottyan LC, Davis BP, Sherrill JD, et al. Genome-wide association analysis of eosinophilic esophagitis provides insight into the tissue specificity of this allergic disease. Nat Genet. 2014; 46:895-900. [PubMed: 25017104]

10. Sleiman PM, Wang ML, Cianferoni A, et al. GWAS identifies four novel eosinophilic esophagitis loci. Nat Commun. 2014; 5:5593. [PubMed: 25407941]

11. Jensen ET, Kuhl J, Martin LJ, et al. Gene-environment Interaction in Pediatric Eosinophilic Esophagitis. Am J Gastroenterol. 2016; 111(Suppl 1)

12. Dellon ES, Kim HP, Sperry SL, et al. A phenotypic analysis shows that eosinophilic esophagitis is a progressive fibrostenotic disease. Gastrointest Endosc. 2014; 79:577-85.e4. [PubMed: 24275329]

13. Singla MB, Chehade M, Brizuela D, et al. Early Comparison of Inflammatory vs. Fibrostenotic Phenotype in Eosinophilic Esophagitis in a Multicenter Longitudinal Study. Clin Transl Gastroenterol. 2015; 6:e132. [PubMed: 26680264]

14. Atkins D, Furuta GT, Liacouras CA, et al. Eosinophilic esophagitis phenotypes: Ready for prime time? Pediatr Allergy Immunol. 2017; 28:312-319. [PubMed: 28339136]

15. Runge TM, Eluri S, Cotton CC, et al. Outcomes of Esophageal Dilation in Eosinophilic Esophagitis: Safety, Efficacy, and Persistence of the Fibrostenotic Phenotype. Am J Gastroenterol. 2016; 111:206-13. [PubMed: 26753894]

16. Lipka S, Boyce HW, Kumar A, et al. The changing faces of eosinophilic esophagitis: the impact of consensus guidelines at the University of South Florida. Dig Dis Sci. 2015; 60:1572-8. [PubMed: 25618310]

17. Eluri S, Runge TM, Cotton CC, et al. The extremely narrow-caliber esophagus is a treatmentresistant subphenotype of eosinophilic esophagitis. Gastrointest Endosc. 2016; 83:1142-8. [PubMed: 26608127]

18. Aceves SS, Newbury RO, Chen D, et al. Resolution of remodeling in eosinophilic esophagitis correlates with epithelial response to topical corticosteroids. Allergy. 2010; 65:109-16. [PubMed: 19796194]

19. Martin LJ, Franciosi JP, Collins MH, et al. Pediatric Eosinophilic Esophagitis Symptom Scores (PEESS v2.0) identify histologic and molecular correlates of the key clinical features of disease. $\mathrm{J}$ Allergy Clin Immunol. 2015; 135:1519-1528 e8. [PubMed: 26051952]

20. Butz BK, Wen T, Gleich GJ, et al. Efficacy, Dose Reduction, and Resistance to High-dose Fluticasone in Patients with Eosinophilic Esophagitis. Gastroenterology. 2014; 147:324-33.e5. [PubMed: 24768678]

21. Dellon ES, Rusin S, Gebhart JH, et al. A clinical prediction tool identifies cases of eosinophilic esophagitis without endoscopic biopsy: A prospective study. Am J Gastroenterol. 2015; 110:134754. [PubMed: 26303128] 
22. Dellon ES, Rusin S, Gebhart JH, et al. Utility of a non-invasive serum biomarker panel for diagnosis and monitoring of EoE: A prospective study. Am J Gastroenterol. 2015; 110:821-7. [PubMed: 25781367]

23. Dellon ES, Cotton CC, Gebhart JH, et al. Accuracy of the Eosinophilic Esophagitis Endoscopic Reference Score in Diagnosis and Determining Response to Treatment. Clin Gastroenterol Hepatol. 2016; 14:31-9. [PubMed: 26404868]

24. Dellon ES, Gebhart JH, Higgins LL, et al. The esophageal biopsy "pull" sign: a highly specific and treatment-responsive endoscopic finding in eosinophilic esophagitis (with video). Gastrointest Endosc. 2016; 83:92-100. [PubMed: 26142556]

25. Wen T, Dellon ES, Moawad FJ, et al. Transcriptome analysis of proton pump inhibitor-responsive esophageal eosinophilia reveals proton pump inhibitor-reversible allergic inflammation. J Allergy Clin Immunol. 2015; 135:187-97. [PubMed: 25441638]

26. Gonsalves N, Policarpio-Nicolas M, Zhang Q, et al. Histopathologic variability and endoscopic correlates in adults with eosinophilic esophagitis. Gastrointest Endosc. 2006; 64:313-9. [PubMed: 16923475]

27. Dellon ES, Speck O, Woodward K, et al. Distribution and variability of esophageal eosinophilia in patients undergoing upper endoscopy. Mod Pathol. 2015; 28:383-90. [PubMed: 25216228]

28. Dellon ES, Fritchie KJ, Rubinas TC, et al. Inter- and intraobserver reliability and validation of a new method for determination of eosinophil counts in patients with esophageal eosinophilia. Dig Dis Sci. 2010; 55:1940-9. [PubMed: 19830560]

29. Rusin S, Covey S, Perjar I, et al. Determination of esophageal eosinophil counts and other histologic features of eosinophilic esophagitis by pathology trainees is highly accurate. Hum Pathol. 2017; 62:50-55. [PubMed: 28041975]

30. Dellon ES, Yellore V, Andreatta M, et al. A single biopsy is valid for genetic diagnosis of eosinophilic esophagitis regardless of tissue preservation or location in the esophagus. $\mathbf{J}$ Gastrointestin Liver Dis. 2015; 24:151-7. [PubMed: 26114173]

31. Wilkerson MD, Hayes DN. ConsensusClusterPlus: a class discovery tool with confidence assessments and item tracking. Bioinformatics. 2010; 26:1572-3. [PubMed: 20427518]

32. Schoepfer AM, Safroneeva E, Bussmann C, et al. Delay in diagnosis of eosinophilic esophagitis increases risk for stricture formation in a time-dependent manner. Gastroenterology. 2013; 145:1230-1236 e2. [PubMed: 23954315]

33. Lipka S, Kumar A, Richter JE. Impact of Diagnostic Delay and Other Risk Factors on Eosinophilic Esophagitis Phenotype and Esophageal Diameter. J Clin Gastroenterol. 2016; 50:134-40. [PubMed: 25710524]

34. Cheng E, Souza RF, Spechler SJ. Tissue remodeling in eosinophilic esophagitis. Am J Physiol Gastrointest Liver Physiol. 2012; 303:G1175-87. [PubMed: 23019192]

35. Dellon ES, Hirano I. Epidemiology and natural history of eosinophilic esophagitis. Gastroenterology. 2017 In press.

36. Kwiatek MA, Hirano I, Kahrilas PJ, et al. Mechanical properties of the esophagus in eosinophilic esophagitis. Gastroenterology. 2011; 140:82-90. [PubMed: 20858491]

37. Nicodeme F, Hirano I, Chen J, et al. Esophageal Distensibility as a Measure of Disease Severity in Patients With Eosinophilic Esophagitis. Clin Gastroenterol Hepatol. 2013; 11:1101-1107.e1. [PubMed: 23591279]

38. Blanchard C, Mingler MK, McBride M, et al. Periostin facilitates eosinophil tissue infiltration in allergic lung and esophageal responses. Mucosal Immunol. 2008; 1:289-96. [PubMed: 19079190]

39. Balastik M, Ferraguti F, Pires-da Silva A, et al. Deficiency in ubiquitin ligase TRIM2 causes accumulation of neurofilament light chain and neurodegeneration. Proc Natl Acad Sci U S A. 2008; 105:12016-21. [PubMed: 18687884]

40. Tocchini C, Ciosk R. TRIM-NHL proteins in development and disease. Semin Cell Dev Biol. 2015; 47-48:52-9. [PubMed: 26514622]

41. Khazaei MR, Bunk EC, Hillje AL, et al. The E3-ubiquitin ligase TRIM2 regulates neuronal polarization. J Neurochem. 2011; 117:29-37. [PubMed: 20796172]

42. Thompson S, Pearson AN, Ashley MD, et al. Identification of a novel Bcl-2-interacting mediator of cell death (Bim) E3 ligase, tripartite motif-containing protein 2 (TRIM2), and its role in rapid 
ischemic tolerance-induced neuroprotection. J Biol Chem. 2011; 286:19331-9. [PubMed: 21478148]

43. Ylikallio E, Poyhonen R, Zimon M, et al. Deficiency of the E3 ubiquitin ligase TRIM2 in earlyonset axonal neuropathy. Hum Mol Genet. 2013; 22:2975-83. [PubMed: 23562820]

44. Schonrock N, Humphreys DT, Preiss T, et al. Target gene repression mediated by miRNAs miR-181c and miR-9 both of which are down-regulated by amyloid-beta. J Mol Neurosci. 2012; 46:324-35. [PubMed: 21720722]

45. Sherrill JD, Kc K, Wu D, et al. Desmoglein-1 regulates esophageal epithelial barrier function and immune responses in eosinophilic esophagitis. Mucosal Immunol. 2014; 7:718-29. [PubMed: 24220297]

46. Hirano I, Dellon ES, Hamilton JD, et al. Dupilumab Efficacy and Safety in Adult Patients With Active Eosinophilic Esophagitis: A Randomized Double-Blind Placebo-Controlled Phase 2 Trial. Am J Gastroenterol. 2017; 112(Suppl 1) AB 20 (ACG 2017).

47. Nilsson SC, Sim RB, Lea SM, et al. Complement factor I in health and disease. Mol Immunol. 2011; 48:1611-20. [PubMed: 21529951]

48. Collins MH, Blanchard C, Abonia JP, et al. Clinical, pathologic, and molecular characterization of familial eosinophilic esophagitis compared with sporadic cases. Clin Gastroenterol Hepatol. 2008; 6:621-9. [PubMed: 18434257]

49. Fahey LM, Guan S, Liacouras CA, et al. Food Allergen Triggers are Increased in Children with the TSLP Risk Allele and Eosinophilic Esophagitis. Gastroenterology. 2017; 152(Suppl):S854-S855. (Tu1082).

50. Eluri S, Selitsky SR, Parker JS, et al. Clinical and Molecular Predictors of Histologic Response to Topical Steroid Treatment in Eosinophilic Esophagitis: Results from a Prospective Study. Gastroenterology. 2017; 152(Suppl):S858. (Tu1092). 


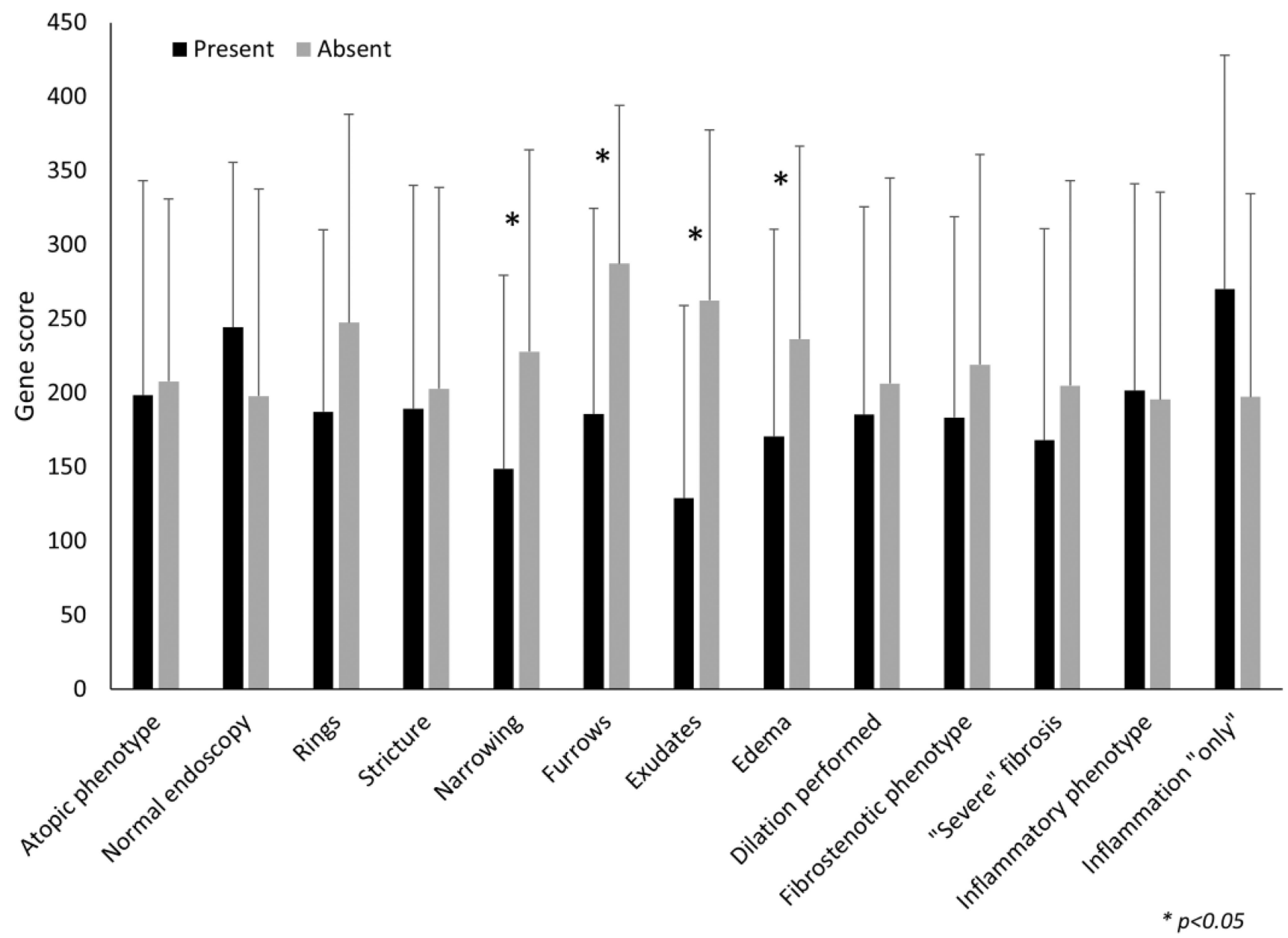

Figure 1.

Gene score by clinical phenotype, endoscopic findings, and endoscopic phenotypes. Black bars indicate the presence of a finding or phenotype. Gray bars indicate the absence of a finding or phenotype. Asterixes indicate a significant difference $(\mathrm{p}<0.05)$ between mean gene scores in EoE cases where the finding/phenotype is present or absent. 


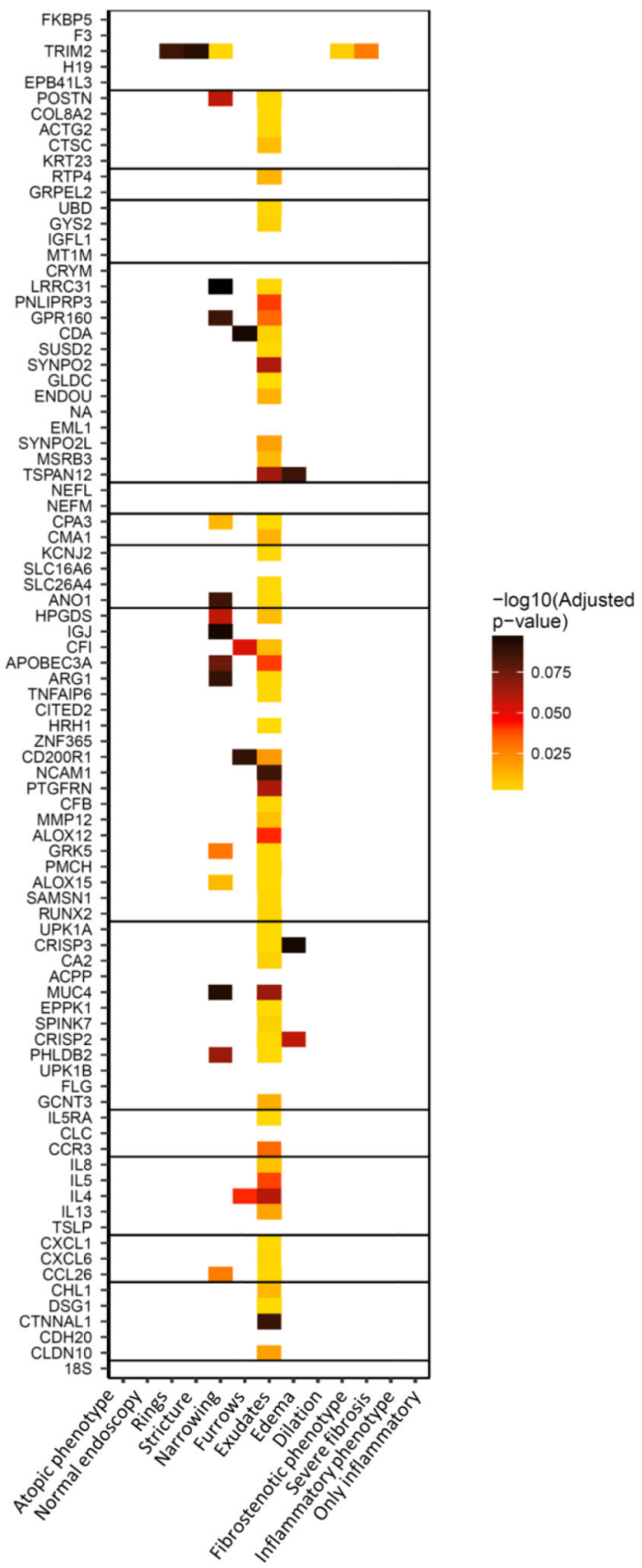

Figure 2.

Heat map showing adjusted $-\log 10$ (p-value) determined by differential expression of the different phenotypes by the Mann-Whitney $U$ test. A tile is displayed if the adjusted q-value $<0.1$ for each gene in the 94 gene panel (noted on the vertical axis), as distributed among clinical phenotype, endoscopic findings, and endoscopic phenotypes (noted on the horizontal axis). The lighter and more yellow the color, the stronger the differential gene expression between EoE cases with or without a finding/phenotype. 
Dellon et al.

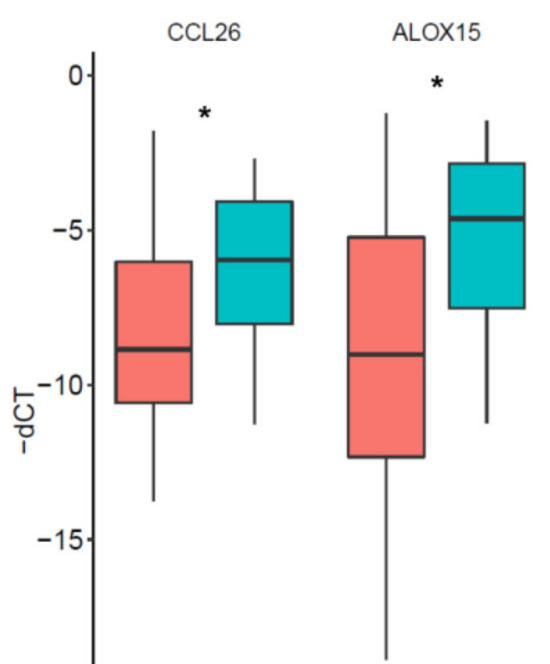

GRK5

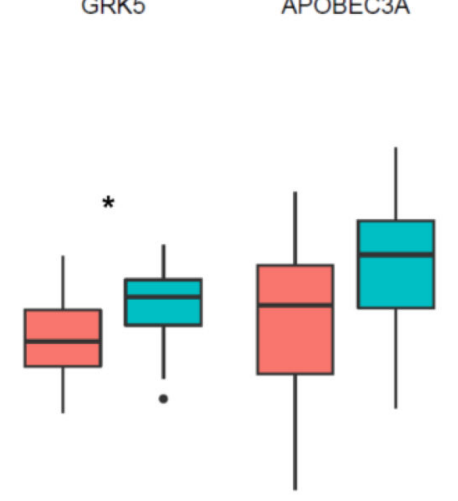

Page 14

HPGDS CPA3 POSTN

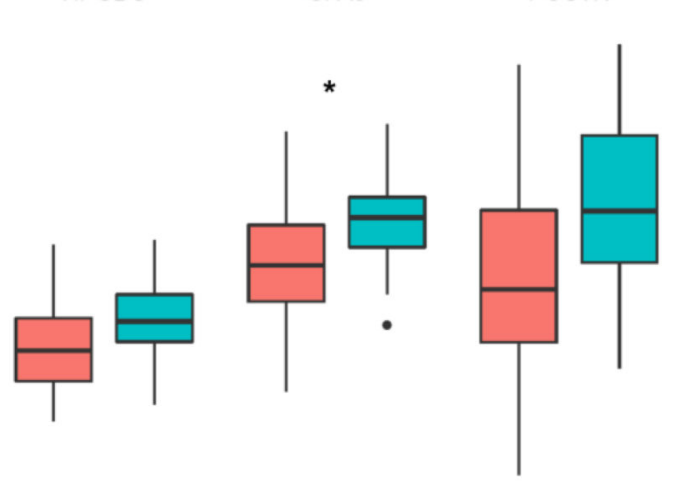

${ }^{*} p<0.05$

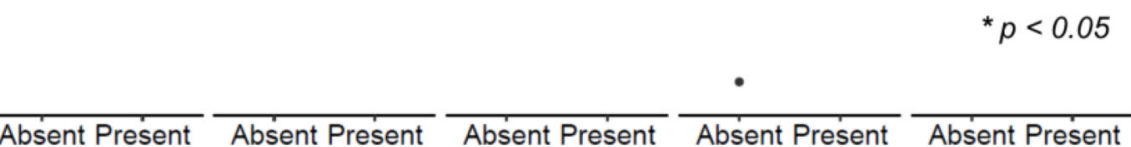

Esophageal narrowing 

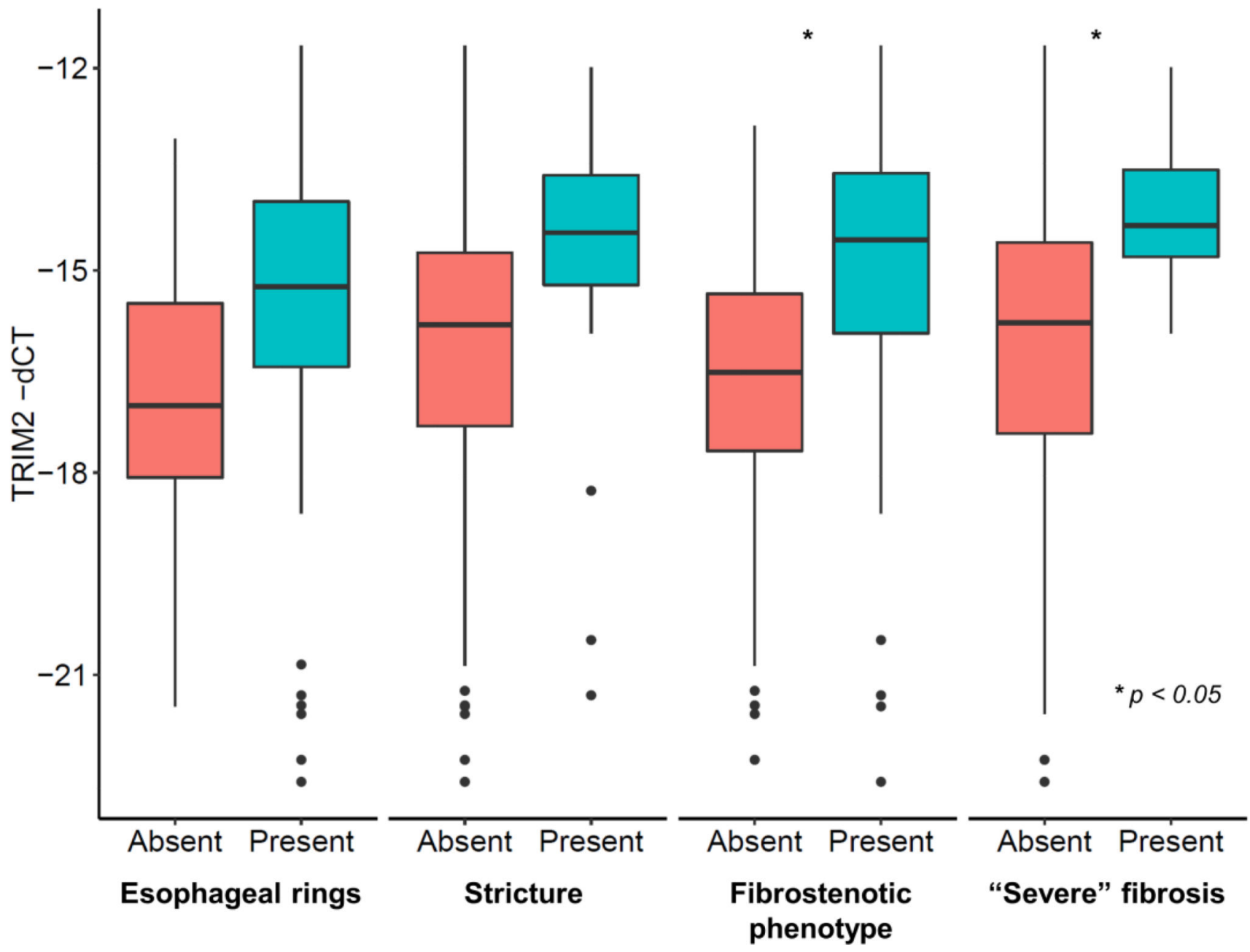

Figure 3.

Boxes represent median \pm interquartile range and whiskers represent $\pm 1.5 *$ interquartile range. qRT-PCR normalized to GAPDH. (A) RNA expression differences in selected genes in patients where esophageal narrowing is either absent (pink) or present (blue). (B) TRIM2 RNA expression differences by selected endoscopic findings and phenotypes, as noted on the y axis. 

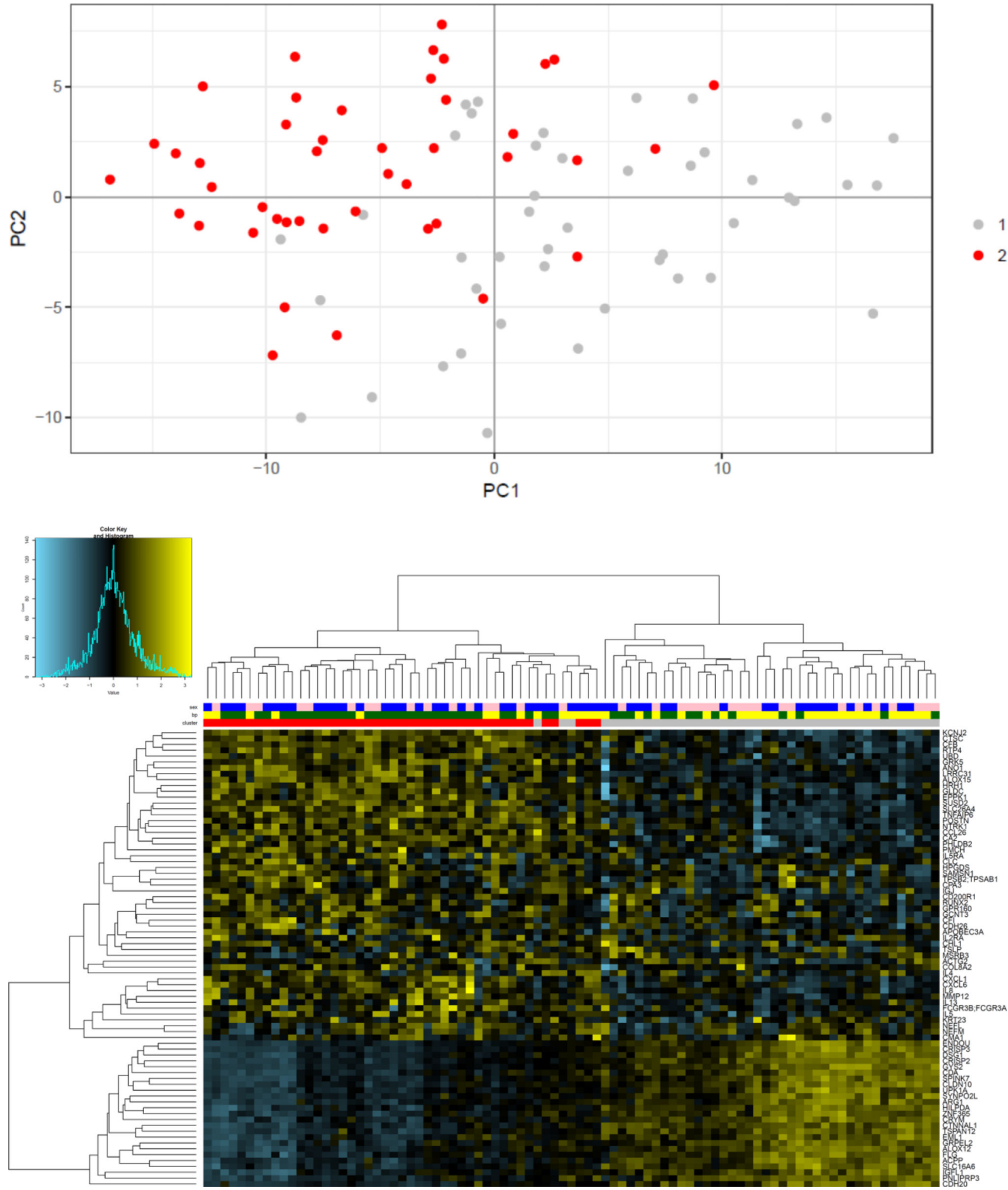

Figure 4.

(A) PC1 and PC2 from a principal component analysis. The color represents the clusters determined by ConsensusClusterPlus used on all genes in the 94 gene panel. This shows that 
the EoE cases independently cluster into two groups (cluster 1 indicated by gray dots and cluster 2 as indicated by red dots) based on gene expression. (B) Heat map showing differential gene expression for all genes (rows) and patient samples (columns) in the study. Samples and genes clustered using hierarchical clustering of Euclidean distance. Decreased gene expression is indicated by more blue coloration and increased expression is indicated by more yellow coloration. The three color bars at the top of the heat map show sex (male in blue; female in pink), presence of exudates (present is yellow; absent is green), and cluster assignment (cluster 1 is gray; cluster 2 cases is red). 


\section{Table 1}

Characteristics and phenotypes of EoE cases after stratification by cluster determined by principle component analysis

\begin{tabular}{|c|c|c|c|}
\hline & $\begin{array}{c}\text { Cluster } 1 \\
(n=45)\end{array}$ & $\begin{array}{c}\text { Cluster } 2 \\
(n=44)\end{array}$ & $\mathbf{P}$ \\
\hline Age (mean years $\pm \mathrm{SD})$ & $36.4 \pm 11.2$ & $38.1 \pm 14.3$ & 0.55 \\
\hline Male (n, \%) & $23(51)$ & $29(66)$ & 0.16 \\
\hline White (n, \%) & $41(91)$ & $43(98)$ & 0.18 \\
\hline \multicolumn{4}{|l|}{ Symptoms (n, \%) } \\
\hline Dysphagia & $44(98)$ & $44(100)$ & 0.32 \\
\hline$>5$ yrs $(\mathrm{n}=70)$ & $16(50)$ & $24(63)$ & 0.27 \\
\hline Heartburn & $7(15)$ & $4(9)$ & 0.35 \\
\hline Abdominal pain & $6(13)$ & $1(2)$ & 0.05 \\
\hline Nausea/vomiting & $1(2)$ & $0(0)$ & 0.32 \\
\hline Any atopic disease (n, \%) & $33(73)$ & $33(75)$ & 0.98 \\
\hline Asthma & $17(38)$ & $9(20)$ & 0.06 \\
\hline Rhinitis/sinusitis & $28(62)$ & $30(68)$ & 0.65 \\
\hline Dermatitis & $5(11)$ & $2(5)$ & 0.24 \\
\hline Food allergies & $18(40)$ & $16(36)$ & 0.66 \\
\hline \multicolumn{4}{|l|}{ Endoscopic findings (n, \%) } \\
\hline Rings & $37(82)$ & $34(77)$ & 0.56 \\
\hline Stricture & $10(22)$ & $12(27)$ & 0.58 \\
\hline Narrowing & $17(38)$ & $15(34)$ & 0.72 \\
\hline Furrows & $41(91)$ & $36(82)$ & 0.20 \\
\hline Crêpe-paper mucosa & $5(11)$ & $0(0)$ & 0.02 \\
\hline White plaques/exudates & $31(69)$ & $11(25)$ & $<0.001$ \\
\hline Edema/decreased vascularity & $30(67)$ & $20(45)$ & 0.04 \\
\hline Hiatal hernia & $5(11)$ & $4(9)$ & 0.75 \\
\hline Dilation performed & $15(33)$ & $14(32)$ & 0.88 \\
\hline Fibrostenotic & $24(53)$ & $25(57)$ & 0.75 \\
\hline "Severe fibrosis" & $6(13)$ & $7(16)$ & 0.73 \\
\hline Inflammatory & $29(64)$ & $28(64)$ & 0.94 \\
\hline "Inflammation only" & $3(7)$ & $3(7)$ & 0.98 \\
\hline Eosinophil count (max eos/hpf \pm SD) & $117.8 \pm 141.1$ & $95.4 \pm 87.6$ & 0.001 \\
\hline \multicolumn{4}{|l|}{ Associated histologic findings (n, \%) } \\
\hline Eosinophil degranulation & $45(100)$ & $40(91)$ & 0.04 \\
\hline Eosinophil microabscesses & $36(80)$ & $20(45)$ & $<0.001$ \\
\hline Basal layer hyperplasia & $24(53)$ & $14(33)$ & 0.02 \\
\hline Spongiosis & $39(87)$ & $36(82)$ & 0.23 \\
\hline
\end{tabular}

Dig Liver Dis. Author manuscript; available in PMC 2019 August 01. 